\title{
sciendo
}

\section{Taekwondo Anaerobic Intermittent Kick Test: Discriminant Validity and an Update with the Gold-Standard Wingate Test}

\author{
by \\ Amel Tayech 1,2, Mohamed Arbi Mejrit,2, Mehdi Chaouachi ${ }^{1,3}$, Helmi Chaabene ${ }^{4,5}$, \\ Mourad Hambli', Matt Brughelli, David G. Behm ${ }^{7}$, Anis Chaouachi ${ }^{1,7}$
}

The aim of this study was to update the validity of the Taekwondo Anaerobic Intermittent Kick Test compared with the 30-s Wingate anaerobic test as the "Gold-Standard", squat jump and countermovement jump tests. The second objective was to examine whether this new specific test would be able to effectively discriminate between elite taekwondo athletes of different competitive levels. Twenty taekwondo athletes (15 males and 5 females) participated in the validation component, whereas 18 (14 males and 4 females) and 16 (13 males and 3 females) athletes participated in the reliability analysis of the Wingate anaerobic test and jumping tests, respectively. They performed these tests on two separate occasions (i.e., test-retest), in addition to the Taekwondo Anaerobic Intermittent Kick Test. To establish test's discriminatory capability (i.e., construct validity), two subgroups were identified based on their international and national taekwondo performance: 10 elite ( 8 males and 2 females) and 9 sub-elite ( 7 males and 2 females) athletes. Wingate anaerobic test and jumping tests performances showed excellent reliability (ICC $>0.90$, SEM $<5 \%$ for most variables). Significant correlations between Taekwondo Anaerobic Intermittent Kick Test, Wingate anaerobic test, and jumping tests' variables were mostly "large". Elite taekwondo athletes showed greater taekwondo test performances compared with their sub-elite counterparts $(p<0.001)$. Receiving operating characteristic analysis indicated that the taekwondo specific test was able to effectively discriminate between elite and sub-elite taekwondo athletes. Overall, the findings of the current study support the concurrent validity of the Taekwondo Anaerobic Intermittent Kick Test. In particular, the Taekwondo Anaerobic Intermittent Kick Test showed good ability to effectively discriminate between taekwondo athletes of different competitive levels.

Key words: muscle power, sport-specific performance, anaerobic power, sensitivity, reliability, striking combat sports.

\section{Introduction}

Taekwondo is an official Olympic sport since 2000. Today, taekwondo is one of the most systematic and scientific Korean traditional martial arts (World Taekwondo, 2018). From a physical fitness standpoint, aerobic and anaerobic power, muscular strength and power, flexibility, speed, and agility represent key components in taekwondo athletes, which are associated with sporting success (Bridge et al., 2014; Chaabene et al., 2018). In particular, taekwondo athletes should acquire high peak anaerobic power, especially in the lower limbs, to perform many sequences of fast and powerful attacks and

\footnotetext{
1 - Tunisian Research Laboratory "Sports Performance Optimization", National Center of Medicine and Science in Sports (CNMSS), Tunis, Tunisia.

2 - High Institute of Sport and Physical Education, Ksar-Saïd, Manouba University, Tunis, Tunisia.

3 - Movement Sport and Health Sciences Laboratory, University of Rennes 2-ENS Cachan, Rennes, France.

4 - Division of Training and Movement Sciences, Research Focus Cognition Sciences, University of Potsdam, Am Neuen Palais 10, 14469 Potsdam, Germany.

5 - High Institute of Sports and Physical Education, Kef, University of Jendouba, Tunisia.

6 - AUT University, Sports Performance Research Institute New Zealand, Auckland, New Zealand.

7 - School of Human Kinetics and Recreation, Memorial University of Newfoundland, St. John's, Newfoundland, Canada.
} 
counter-attacks (Bouhlel et al., 2006), with short (incomplete) recovery periods (Bridge et al., 2014). In this context, Bridge et al. (2014) have reported that these attributes appear to be conducive to achieving success in international competition. Recently, several taekwondo-related studies have focused on the development of sport-specific tests that meet the particular demands of the discipline (Araujo et al., 2017; Chaabene et al., 2018; Rocha al., 2016a,b; Tayech et al., 2018). Against this background and as part of the assessment of anaerobic power in taekwondo athletes, Tayech et al. (2018) recently studied the reliability and criterion validity of the Taekwondo Anaerobic Intermittent Kick Test (TAIKT). The same authors reported that TAIKT's performance characteristics and physiological strain were comparable to an authentic taekwondo match. In fact, the TAIKT was designed to evaluate anaerobic power, while determining absolute and relative peak power (Ppeak) and mean power (Pmean), as well as the fatigue index (FI). Based on the study results of Tayech et al. (2018), the TAIKT presented good absolute (i.e., the standard error of measurement (SEM) was relatively "low", the smallest worthwhile change (SWC) was slightly higher than SEM, thereby demonstrating that the ability of the TAIKT to detect a change was rated as "good") and relative (i.e., intraclass correlation coefficient (ICC) between the test and retest was $>0.90$ ) reliability. Moreover, the TAIKT showed clear and good criterion (concurrent) validity when compared with the Running-based Anaerobic Sprint Test (Tayech et al., 2018). In view of these results, Tayech et al. (2018) reported that the advantage of using the TAIKT to measure the anaerobic power of taekwondo athletes was that it allowed the execution of movements more specific to taekwondo, such as kicking as their principal technique during the attack and counterattack sequences (i.e., high-intensity intermittent kick techniques). Similarly, Bridge et al. (2014) affirmed that taekwondo athletes should have high anaerobic capabilities to effectively manage the energy needs of combat.

The 30-s Wingate anaerobic test (WAnT) has been shown to be a valid and reliable tool for the assessment of anaerobic power and functional performance of elite athletes (Bar-Or, 1987). Furthermore, this test is the most common method for assessing maximal anaerobic power and capacity among taekwondo athletes (Bridge et al., 2014). However, to date, data related to Ppeak and Pmean of Tunisian elite taekwondo athletes using the WAnT are still missing. Likewise, jumping tests (e.g., Abalakov, squat jump (SJ), countermovement jump (CMJ)) are generally used to evaluate lower limb anaerobic (mechanical) power amongst taekwondo athletes (Bridge et al., 2014; Chaabene et al., 2018). Indeed, significant correlations have been reported between jumping tests (e.g., SJ, CMJ) and taekwondo kick performances (e.g., frequency, force, velocity and power of kicks) (Rocha et al., 2016a; Sant'Ana et al., 2014). In view of the above considerations, the need to verify the validity of this test with the "Gold-Standard" is necessary for its credibility. In this context, Hopkins (2009) indicated that any test should respond to the reliability, validity and sensitivity standard criteria to be scientifically approved. Therefore, this study aimed at examining the validity of the TAIKT compared with the WAnT, SJ and CMJ tests, and to show whether this taekwondo specific test would be sensitive and allow to discriminate between elite taekwondo athletes of different competitive levels (i.e., elite and subelite). We hypothesized that: i) the WAnT, SJ and $\mathrm{CMJ}$ tests would show good reliability among taekwondo athletes, ii) large correlations would be evident between TAIKT and WAnT, SJ, and CMJ variables, and iii) the TAIKT would be able to distinguish between elite and sub-elite Taekwondo athletes.

\section{Methods}

\section{Participants}

Twenty taekwondo athletes including 15 males and 5 females, members of the Tunisian Taekwondo national team volunteered for this study (Table 1). They had $8.1 \pm 2.7$ (mean \pm SD) years of taekwondo training experience. Additionally, they were regularly competing at a national level for $>6$ years and international level for $>2$ years. They were engaged in nine training sessions weekly with two hours each. The experiment was conducted during the competitive phase of the taekwondo season of the year 2017. Eighteen athletes (14 males and 4 females) out of the total sample participated in the reliability analyses of the WAnT (Table 1), and sixteen (13 males and 3 females) of the total 
sample participated in the reliability analyses of SJ and CMJ tests (Table 1). Based on their international and national taekwondo records (i.e., medal winners), 10 athletes were included in the elite ( 8 males and 2 females) and 9 athletes in the sub-elite (7 males and 2 females) subgroups, respectively (Table 1). It is noteworthy that one female from the elite group was randomly excluded to make an equal number of female athletes between the two groups. After a detailed explanation of the experimental protocol, all athletes/legal representatives gave written consent to participate in this study. The study was conducted in accordance with the Declaration of Helsinki and the protocol was fully approved by the Ethics Committee of the National Center of Medicine and Sciences in Sport of Tunisia before the start of the assessments.

\section{Design and Procedures}

The TAIKT is a sport-specific field test designed to evaluate $P_{\text {peak, }} P_{\text {mean, }}$ and FI based on the number of recorded kicks with an electronic body protector. The TAIKT was performed by elite and sub-elite taekwondo athletes to establish its discriminative ability. The reliability of the undertaken tests was established by means of testretest trials separated by one week. Anthropometrical measurements were taken for each participant three days before the start of the experiment. All testing sessions were conducted indoors at the same time of day (i.e., between 4 $\mathrm{pm}$ and $6 \mathrm{pm}$ ), and under the same environmental conditions (i.e., temperature $25^{\circ} \mathrm{C}$ and $63 \%$ humidity) in randomized and counterbalanced order. Tests were preceded by a 15-min standardized warm-up that included jogging, squatting, jumping, and stretching for the SJ and CMJ, and a specific warm-up for the WAnT and TAIKT. The heart rate (HR) was measured every 5 s during each test (Polar Electro Oy, T61-coded, Hungary). The blood lactate concentration [ $\left.\mathrm{La}^{-}\right]$ was recorded using the Lactate Pro Analyzer (Arkray, Tokyo, Japan) 3 min post-test. The rating of perceived exertion (RPE) was recorded immediately after the end of each test (i.e., WAnT and TAIKT) using the 15-point scale, which ranged from 6 (very, very light) to 20 (very, very hard). Verbal encouragement was consistently given to all participants throughout the tests. Participants were instructed to refrain from any strenuous physical effort $24 \mathrm{~h}$ before testing and to avoid eating at least three hours before each testing session.

\section{Taekwondo Anaerobic Intermittent Kick Test (TAIKT)}

As previously described by Tayech et al. (2018), the TAIKT protocol required the athlete to undertake the maximal number of stationary roundhouse kicks (named "Bandal-Tchagui") after the sound signal, alternating right and left legs, during six sets of $5 \mathrm{~s}$, interspersed with $10 \mathrm{~s}$ active recovery (i.e., bouncing movements) between each set. The total time for kicks execution during the TAIKT was $30 \mathrm{~s}$. The kicks were executed on an electronic body protector (TK-Strike Protector, Daedo, Barcelona, Spain) placed around a hanging punching bag, which was stabilized by one of the researchers. The number of kicks was automatically displayed on the computer screen after each kicking set. TAIKT performances were expressed as absolute $(\mathrm{W})$ and relative $\left(\mathrm{W} \cdot \mathrm{kg}^{-0.67}\right)$ $P_{\text {peakTAIKT }}$ and PmeantaikT, and absolute (W) FItAiKt.

\section{0-s Wingate Anaerobic Test (WAnT)}

The WAnT is a 30 -s cycle-ergometer test developed to evaluate an individual's power output (Driss and Vandewalle, 2013). The test was conducted on a friction belt cycle ergometer (Monark 894 E Peak Bike, Weight Ergometer, Vansbro, Sweden, Software version 2.22) with a basket weight loading system interfaced to a microcomputer and fitted with toe clips and heel straps, and individually adjusted for saddle height. Participants had been familiarized with the test protocol on separate occasions prior to collection of definitive data; they performed highvelocity sprint exercises interspersed with $3 \mathrm{~min}$ of rest intervals, so as to minimize continued test learning during the definitive experiment (Attia et al., 2014). Optimal saddle and handlebar positioning was determined for each subject prior to their first test, and the same placement was used in subsequent tests. Toe clips were used throughout (Attia et al., 2014). The test was preceded by a standardized warm-up (Attia et al., 2014; Hachana et al., 2012) that consisted of alternating three 30 -s bouts of exercise at increasing external resistance $(25,50$, and $75 \%$ of the definitive test loading, respectively) and three $30-\mathrm{s}$ periods of active rest (zero-resistance pedalling at $60 \mathrm{rpm})$. The WAnT commenced from a rolling start against minimal resistance and was performed against a constant resistance $(7.5 \%$ 
of the participant's body mass) (Attia et al., 2014; Bar-Or, 1987; Hachana et al., 2012). During the test, participants were instructed to maximally accelerate from a seated posture to avoid the effect of postural changes and to pedal at the maximal effort. All participants were strongly and consistently encouraged throughout the test. Three indices of anaerobic performance expressed as $P_{\text {picWAnT }}$ (highest 5-s output), $P_{\text {meanWAnT (average }}$ power throughout the test), and FIWAnT (decrease in power output from $\mathrm{P}_{\text {picWAnT }}$ to minimal power output $\left.\mathrm{P}_{\min W A n T}\right)$ were calculated according to accepted procedures (Bar-Or, 1987), P PicWAnT and $P_{\text {meanWAnT }}$ being expressed as absolute values (watts) and relative to the $0.67^{\text {th }}$ power of body mass (W·kg-0.67) (Hachana et al., 2012).

\section{Vertical jumping tests}

The jump tests consisted of two vertical jumps (i.e., SJ and CMJ). Before starting the jump tests, participants performed a 15-min standardized warm-up that included accelerations, jumping (Chaouachi et al., 2010) and dynamic stretching (Chaouachi et al., 2017). A 5 -min rest period was provided before starting the tests (Chaouachi et al., 2010). For each jump test, three trials were performed, with $\sim 60 \mathrm{~s}$ of rest inbetween. The best trial was recorded for further analysis. A 3-min rest period was provided between tests (SJ and CMJ) to avoid any fatigue effect. During the SJ as CMJ, hands were kept akimbo to avoid any influence of upper limbs motion on jump performance. Jump performances were assessed with an infrared jump system (Optojump Next instrument, Version 1.3.20.0, Microgate, Bolzano, Italy) interfaced with a microcomputer. This system was developed to measure the flight and ground contact times with $10^{-3}$ s precision, from which the jump height was calculated.

\section{Statistical Analyses}

Two statistical software packages, SPSS 20 (for Windows, Inc., Chicago, IL) and MedCalc (Version 14.8-@1993-2014 medCalc Software bvba), were used for data analyses. Data are presented as mean and standard deviation (mean \pm SD). After normal distribution was examined using the Kolmogorov-Smirnov test, a paired Student $t$-test was used to compare values between the test and retest. An unpaired Student $t$-test was used to compare performances of elite and sub-elite subgroups. Relative reliability was assessed using the intraclass correlation coefficient (ICC) between the test and retest. An ICC $<0.40$ was considered low; between 0.40 and 0.70 acceptable; between 0.70 and 0.90 good and $>0.90$ excellent (Hopkins, 2009). Absolute reliability was analysed by calculating the standard error of measurement (SEM) as follows: $\mathrm{SEM}=\mathrm{SD} \times \sqrt{1}-$ ICC (Stratford, 2004). The usefulness of the test was assessed by calculating the smallest worthwhile change (SWC) as: SWC = 0.2 multiplied by the between subject's standard deviation (Hopkins, 2004). The ability of the test to detect small and meaningful performance changes was rated as good if SEM $\leq$ SWC, satisfactory when SEM $=$ SWC, and marginal in case SEM $\geq$ SWC (Liow and Hopkins, 2003). The minimal detectable change (MDC95\%) at the 95\% confidence interval $(\mathrm{CI})$ of the difference in the score between paired observations was determined as $\mathrm{MDC} 95 \%=\mathrm{SEM} \times \sqrt{2} \times 1.96$ (Stratford, 2004). The stability of the reliability determination was quantified using the $95 \%$ limits of agreement method of Bland and Altman (1986). Effect size $(d z)$ was calculated using GPOWER software (Bonn FRG, Bonn University, Department of Psychology) (Faul and Erdfelder, 2004). Association between TAIKT, WAnT, SJ, and CMJ performances was assessed using the Pearson correlation coefficient $(r)$. The following criteria were adopted for the interpretation of the magnitude of the correlation: trivial $(r<0.1)$, small $(0.1 \leq r<0.3)$, moderate $(0.3 \leq r<0.5)$, large $(0.5 \leq r$ $<0.7)$, very large $(0.7 \leq r<0.9)$, nearly perfect $(0.9 \leq$ $r<1)$ and perfect $(r=1)$ (Hopkins, 2009). Discriminant validity of the TAIKT was analysed using the receiver operator characteristics (ROC) curve (Deyo and Centor, 1986; Mannion et al., 2005) with analyses of the area under the curve (AUC). The ROC curve analysis determines the sensitivity and specificity of a tool to evaluate the ability of the different tests that can discriminate between individuals of different competitive levels. The cut-off value for a good discriminative ability is 0.70 (Deyo and Centor, 1986; Mannion et al., 2005). The significance level was set at $p<0.05$.

\section{Results}

There were no significant differences between the test and retest with respect to the WAnT, SJ, and CMJ (Table 2).

The relative and absolute reliability 
outcomes of the WAnT, SJ, and CMJ are displayed in Table 2. Excellent relative reliability (ICC > 0.90) was observed for the WAnT, SJ, and CMJ. However, for FIwAnT, HR peakWAnT and RPEWAnT, good relative reliability was shown $(\mathrm{ICC}=0.82,0.71$ and 0.82 , respectively). The SEM values for all WAnT variables, the SJ and CMJ were low $(<5 \%)$. The SWC values recorded for all tests were higher than the SEM. Nevertheless for FIwAnT, HR peakWAnT, and RPEWAnT values were rated as marginal. The MDC95\% limits of agreement were acceptable for most of the tests' variables (Table 2).

The other factors that supported the reliability of the WAnT, SJ, and CMJ were BlandAltman plots (Bland and Altman, 1986), which showed excellent results of concordance in all variables (Figure 1). The bias $\pm 95 \%$ limits of agreement for performance and physiological variables of the WAnT were $0.5 \pm 66.96$ for $P_{\text {peakWAnT }}$ (W) (Figure 1A); $-0.1 \pm 4.13$ for $P_{\text {peakWAnT }}\left(\mathrm{W} \cdot \mathrm{kg}^{-0.67}\right) \quad$ (Figure $\left.1 \mathrm{~B}\right) ; 0.4 \pm 27.24$ for PmeanWanT (W) (Figure 1C); $0.0 \pm 1.74$ for $\mathrm{P}_{\text {meanWAnT }}\left(\mathrm{W} \cdot \mathrm{kg}^{-0.67}\right)$ (Figure 1D), $-1.0 \pm 3.07$ for
FIwAnT $\left(\mathrm{W} \cdot \mathrm{s}^{-1}\right)$ (Figure 1E), $0.7 \pm 9.10$ for HReakWAnT (bpm) (Figure 1F) and $-0.2 \pm 1.09$ for RPEwAnT (Figure 1G). The bias $\pm 95 \%$ limits of agreement for $\mathrm{SJ}$ and $\mathrm{CMJ}$ performances were $-1.2 \pm 3.36$ (Figure 1H) and $-4.1 \pm 9.15$ (Figure 1I), respectively.

The correlation coefficient $(r)$, confidence interval (CI) and magnitude of the correlation between TAIKT, WAnT, SJ, and CMJ variables are shown in Table 3. Moderate-to-large correlation coefficients were observed between the TAIKT and the WAnT, SJ, and CMJ.

The TAIKT sport-specific performance (Ppeaktaikt, Pmeantaikt, and FIталкт), physiological

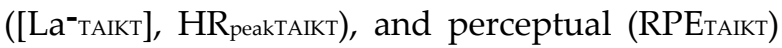
characteristics for elite and sub-elite taekwondo athletes are presented in Table 4. The unpaired sample $t$-test showed significantly higher TAIKT performance in elite compared to sub-elite taekwondo athletes. However, no significant between groups differences were observed for [La-TAIKT], HR peakTAIKT, and RPEтAIKT (Table 4).

Table 1

Characteristics of athletes participating in the different parts of the study

\begin{tabular}{|c|c|c|c|c|c|c|}
\hline $\begin{array}{l}\text { Parts of the } \\
\text { study }\end{array}$ & $\begin{array}{l}\text { Participants } \\
\text { (n) }\end{array}$ & Age (year) & Height (cm) & $\mathrm{BM}(\mathrm{kg})$ & $\mathrm{BMI}\left(\mathrm{kg} \cdot \mathrm{m}^{-2}\right)$ & $\mathrm{BF}(\%)$ \\
\hline Validity of the & \multirow[b]{2}{*}[5\mathrm{F}]{} & $17.5 \pm 0.6$ & $179.7 \pm 7.5$ & $64.1 \pm 7.6$ & $20 \pm 0.4$ & $8 \pm 1.7$ \\
\hline $\begin{array}{l}\text { TAIKT with the } \\
\text { WAnt, SJ and } \\
\text { CMJ }\end{array}$ & & $17.6 \pm 0.5$ & $163.2 \pm 3.9$ & $52.3 \pm 4.6$ & $19.7 \pm 1.9$ & $17.5 \pm 2.67$ \\
\hline $\begin{array}{l}\text { WAnT } \\
\text { reliability }\end{array}$ & $18[14 \mathrm{M}, 4 \mathrm{~F}]$ & $17.4 \pm 0.6$ & $175.4 \pm 9.1$ & $61.3 \pm 8.2$ & $20 \pm 2.3$ & $10.2 \pm 4.6$ \\
\hline $\begin{array}{l}\text { SJ and CMJ } \\
\text { reliability }\end{array}$ & $16[13 \mathrm{M}, 3 \mathrm{~F}]$ & $17.4 \pm 0.6$ & $176.9 \pm 8.4$ & $61.7 \pm 8.6$ & $19.8 \pm 2.4$ & $9.7 \pm 3.8$ \\
\hline \multirow{2}{*}{$\begin{array}{l}\text { Discriminant } \\
\text { validity of the } \\
\text { TAIKT }\end{array}$} & $\begin{array}{l}10 \text { Elite } \\
{[8 \mathrm{M}, 2 \mathrm{~F}]}\end{array}$ & $17.7 \pm 0.7$ & $179.1 \pm 10.5$ & $66 \pm 8.8$ & $20.8 \pm 2.7$ & $10.2 \pm 4.4$ \\
\hline & $\begin{array}{l}9 \text { Sub-elite } \\
{[7 \mathrm{M}, 2 \mathrm{~F}]}\end{array}$ & $17.3 \pm 0.5$ & $172.4 \pm 8.9$ & $56.4 \pm 5.6$ & $19 \pm 1.4$ & $9.6 \pm 4.4$ \\
\hline
\end{tabular}

Values are presented as mean \pm standard deviation.

TAIKT, Taekwondo Anaerobic Intermittent Kick Test; WAnT, 30-s Wingate anaerobic test; SJ, squat jump; CMJ, countermovement jump; $M$, male; $F$, female; $B M$, body mass; $B M I$, body mass index; $B F$, body fat 
Table 2

Descriptive performances of taekwondo athletes, Student's t-test and mean

differences for the test-retest complemented with reliability statistics of the WAnT, SJ and CMJ

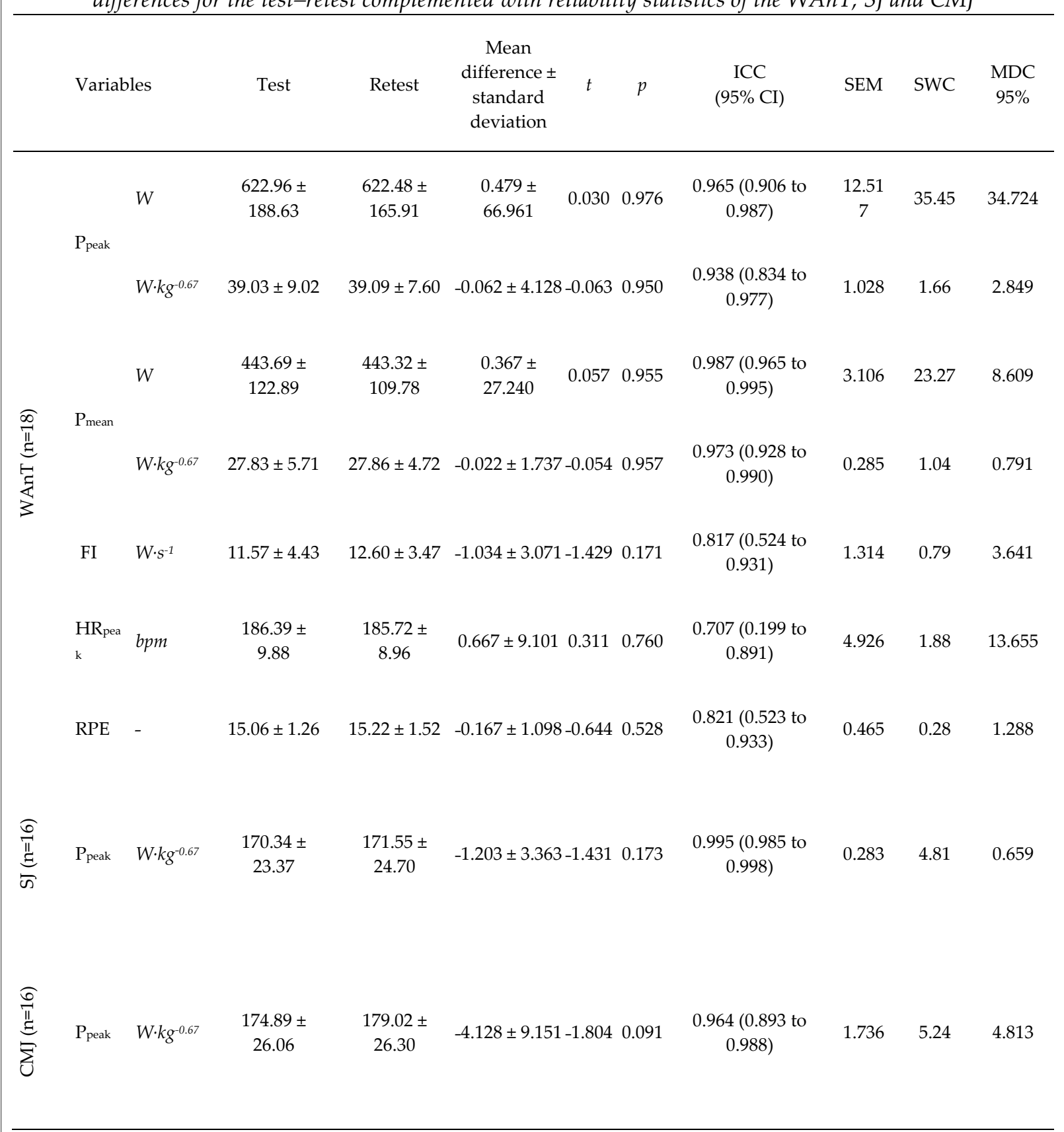

Values are presented as mean \pm standard deviation.

WAnT, 30-s Wingate anaerobic test; SJ, squat jump; CMJ, countermovement jump; $P_{\text {penk, }}$ peak power; $P_{\text {mean, }}$ mean power; FI, fatigue index; HRpeak, peak heart rate; RPE, ratings of perceived exertion; $t$, Student's $t$; $p$, significations; ICC, intraclass-correlation coefficient; CI, confidence interval; SEM, standard error of measurement; SWC, smallest worthwhile change; MDC95\%, minimal detectable change at 95\% confidence interval 
Table 3

Pearson's correlation ( $r$ ) of performances and physiological variables between the TAIKT and the WAnT, SJ and CMJ $(n=20)$

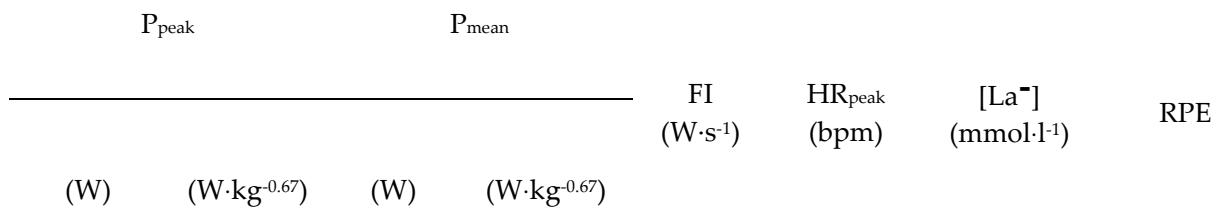

\begin{tabular}{|c|c|c|c|c|c|c|c|}
\hline $\begin{array}{l}\text { Mean } \pm \\
\text { SD }\end{array}$ & $\begin{array}{c}14.57 \pm \\
6.20\end{array}$ & $\begin{array}{c}0.91 \pm \\
0.32\end{array}$ & $\begin{array}{c}9.68 \pm \\
4.43\end{array}$ & $0.60 \pm 0.24$ & $\begin{array}{c}0.30 \pm \\
0.11\end{array}$ & $\begin{array}{c}188.85 \pm \\
9.05\end{array}$ & $\begin{array}{c}10.96 \pm \\
1.59\end{array}$ \\
\hline
\end{tabular}

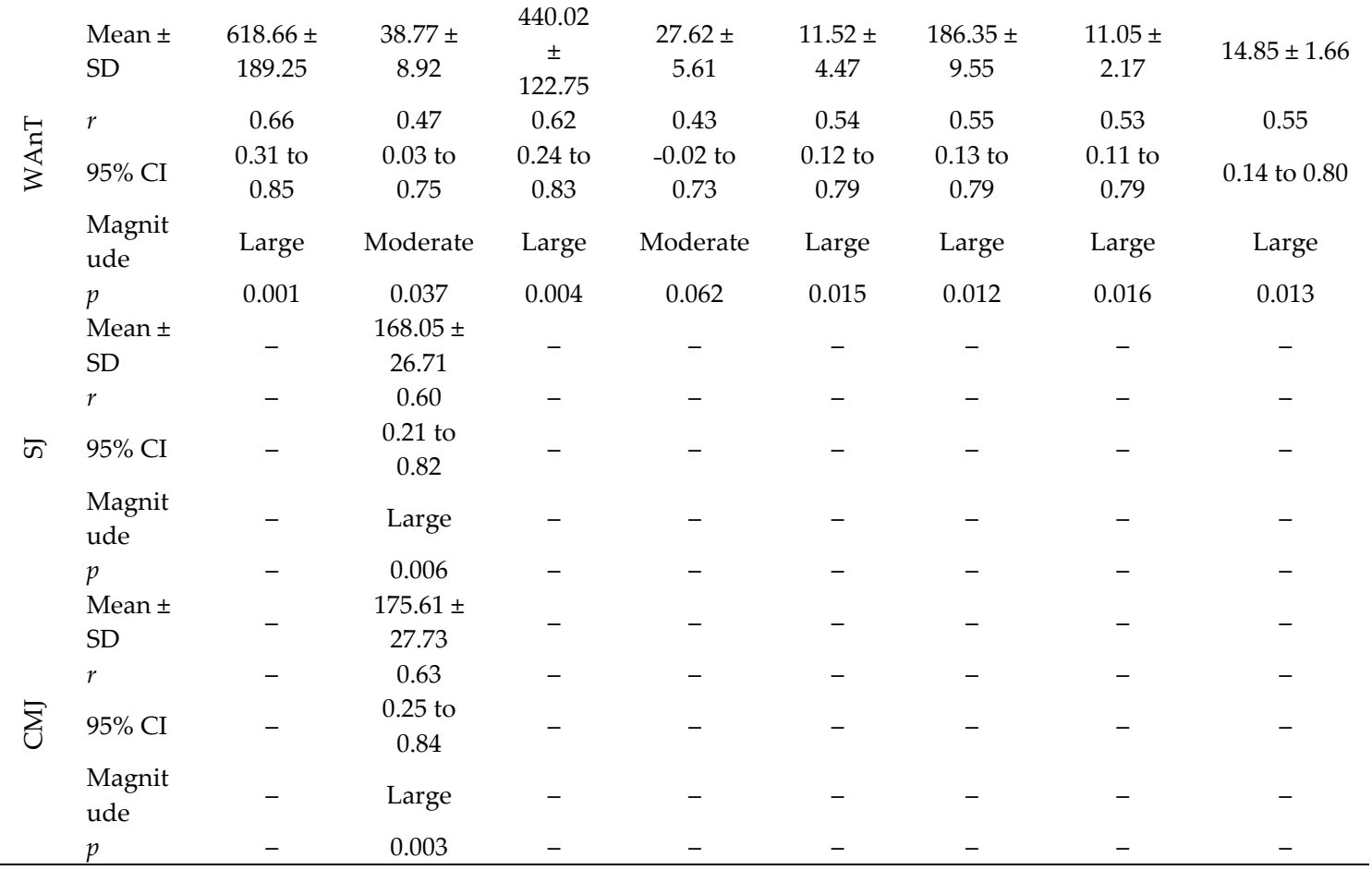

TAIKT, Taekwondo Anaerobic Intermittent Kick Test; WAnT, 30-s Wingate anaerobic test; SJ, squat jump; CMJ, countermovement jump; $P_{\text {peak, }}$ peak power; $P_{\text {mean, }}$ mean power;

FI, fatigue index; HRpeak, peak heart rate; [La-], lactate concentration; RPE, ratings of perceived exertion; SD, standard deviation; $r$, Pearson correlation coefficient; $C I$, confidence interval. 


\section{Table 4}

Comparison of performances, physiological and perceptual variables between elite and sub-elite taekwondo athletes during and after the TAIKT

\begin{tabular}{|c|c|c|c|c|c|c|c|}
\hline \multicolumn{2}{|c|}{ Variables } & $\begin{array}{c}\text { Elite } \\
(\mathrm{n}=10)\end{array}$ & $\begin{array}{l}\text { Sub-elite } \\
(\mathrm{n}=9)\end{array}$ & $\begin{array}{c}\text { Mean difference } \\
\pm \text { standard } \\
\text { deviation }\end{array}$ & $t$ & $p$ & $d z$ \\
\hline & $W$ & $18.98 \pm 5.06$ & $10.72 \pm 3.23$ & $8.260 \pm 1.975$ & 4.183 & 0.0006 & -1.92 \\
\hline \multicolumn{8}{|l|}{$P_{\text {peak }}$} \\
\hline & $W \cdot \mathrm{kg}^{-0.67}$ & $1.14 \pm 0.24$ & $0.72 \pm 0.21$ & $0.420 \pm 0.102$ & 4.119 & 0.0007 & -1.86 \\
\hline & $W$ & $12.98 \pm 3.30$ & $6.83 \pm 2.22$ & $6.147 \pm 1.307$ & 4.704 & 0.0002 & -2.16 \\
\hline \multicolumn{8}{|l|}{ Pmean } \\
\hline & $W \cdot \mathrm{kg}^{-0.67}$ & $0.78 \pm 0.16$ & $0.46 \pm 0.15$ & $0.321 \pm 0.072$ & 4.466 & 0.0003 & -2.06 \\
\hline FI & $W \cdot s^{-1}$ & $0.36 \pm 0.11$ & $0.25 \pm 0.09$ & $0.110 \pm 0.045$ & 2.423 & 0.027 & -1.09 \\
\hline $\mathrm{HR}_{\text {peak }}$ & bpm & $187.80 \pm 7.87$ & $192.22 \pm 7.82$ & $-4.422 \pm 3.606$ & -1.226 & 0.237 & 0.563 \\
\hline$\left[\mathrm{La}^{-}\right]$ & $\mathrm{mmol} \cdot \mathrm{l}^{-1}$ & $11.24 \pm 1.61$ & $10.84 \pm 1.60$ & $0.396 \pm 0.737$ & 0.536 & 0.599 & -0.25 \\
\hline RPE & - & $13.60 \pm 1.07$ & $14.00 \pm 0.71$ & $-0.400 \pm 0.423$ & -0.946 & 0.357 & 0.436 \\
\hline
\end{tabular}

TAIKT, Taekwondo Anaerobic Intermittent Kick Test; $P_{\text {peak, }}$ peak power; $P_{\text {mean, }}$ mean power; FI, fatigue index; HRpeak, peak heart rate; [La-], lactate concentration; RPE, ratings of perceived exertion; $t$, Student's $t ; p$, significance; $d z$, Effect size 

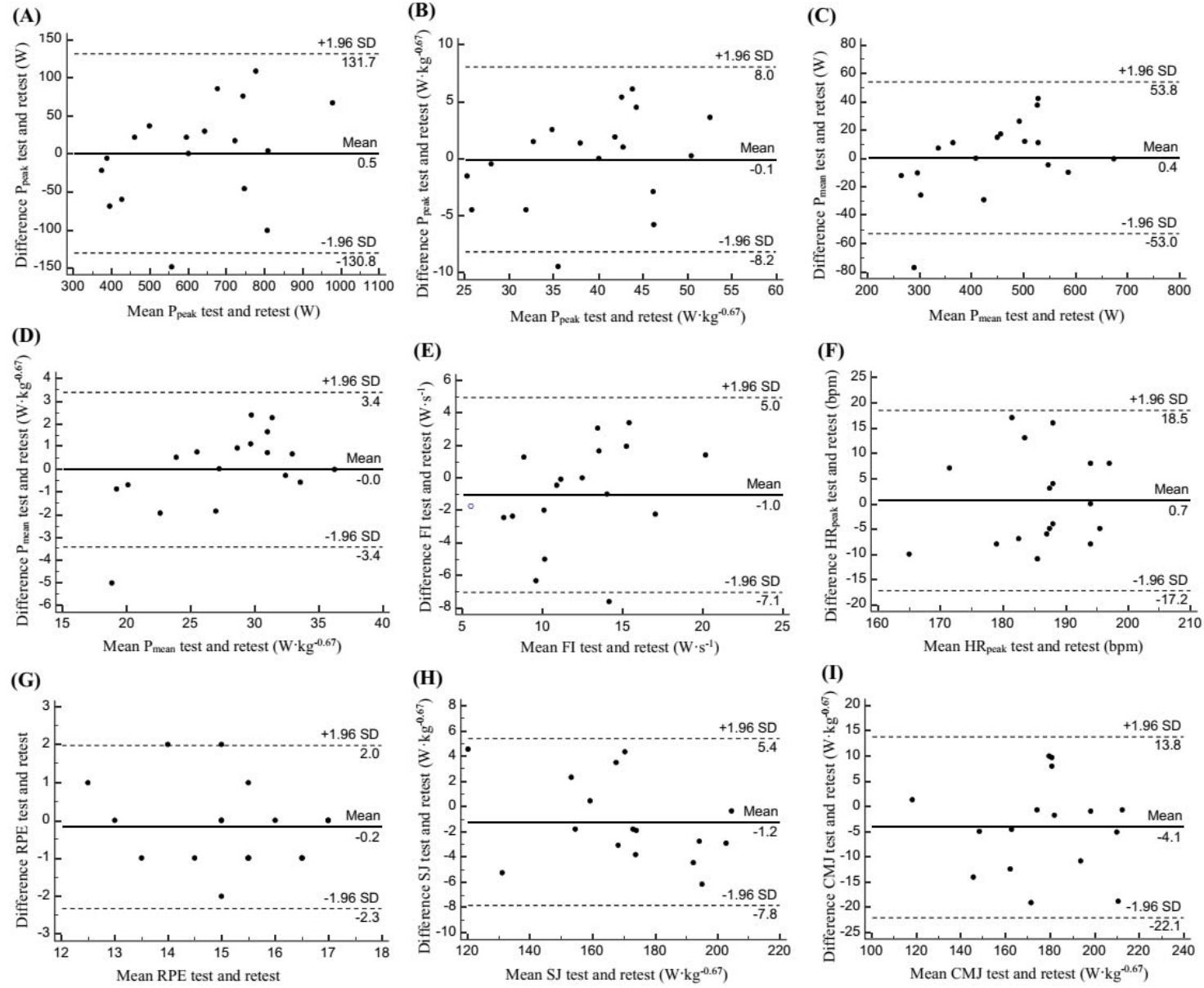

Figure 1

Reliability analysis of the physical tests verified by Bland-Altman plots of the WAnT, SJ and $C M J$ test-retest

(A): $P_{\text {peak }}(W) ;(B): P_{\text {peak }}\left(W \cdot \mathrm{kg}^{-0.67)}\right) ;(C): P_{\text {mean }}(W) ;(D): P_{\text {mean }}\left(W \cdot \mathrm{kg}^{-0.67}\right),(E): F I\left(W \cdot \mathrm{s}^{-1}\right)$; (F):HRpeak (bpm); (G): RPE; (H): SJ; (I): CMJ. WAnT: 30-s Wingate anaerobic test; SJ: squat jump; CMJ: countermovement jump. Upper and lower horizontal dotted lines represent the limits of agreement (mean \pm 1.96 SD of the difference between the test and retest). 

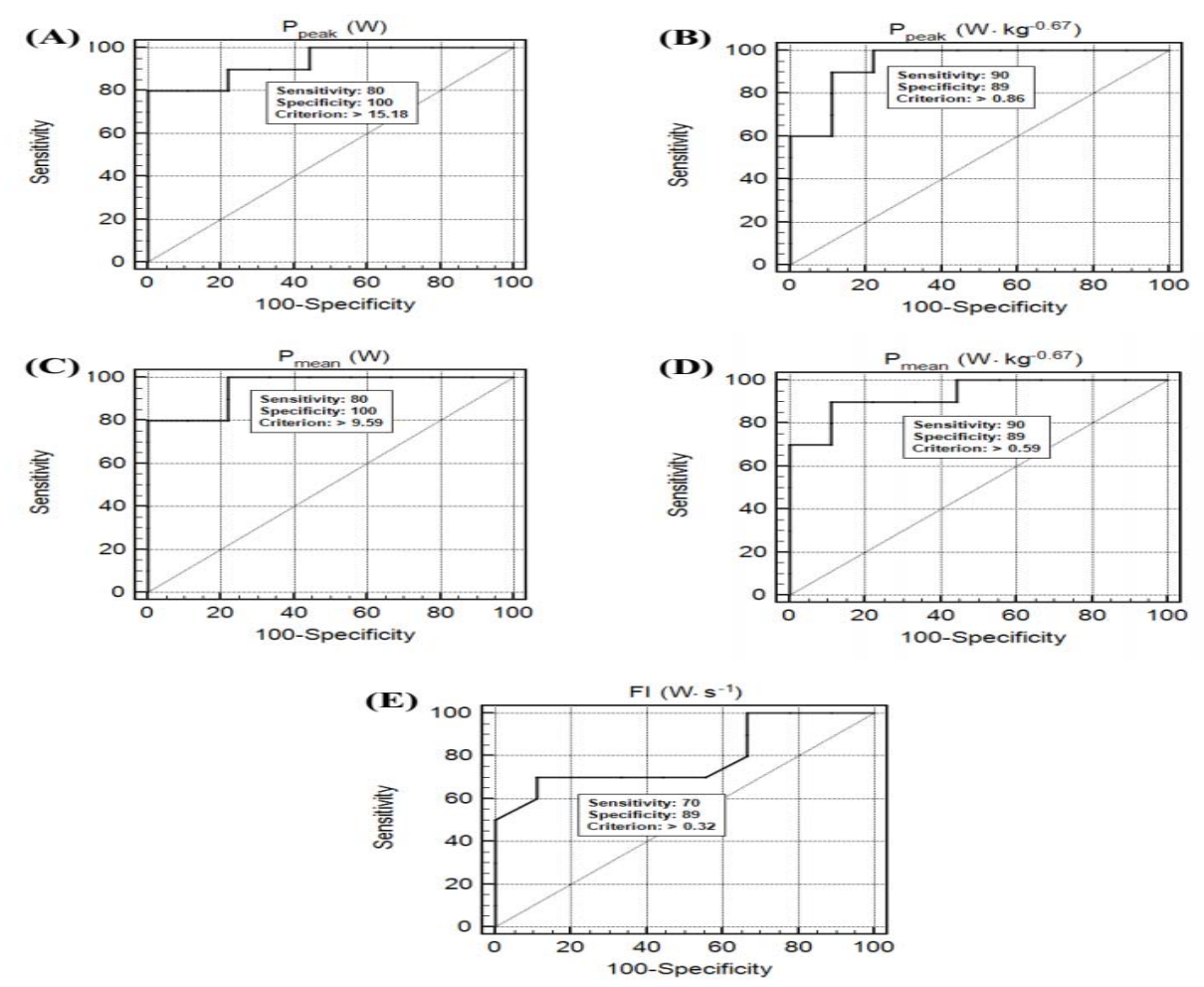

Figure 2

Receiver operating characteristics (ROC) curve for the TAIKT performances for elite and sub-elite taekwondo athletes

TAIKT: Taekwondo Anaerobic Intermittent Kick Test; $(A): P_{\text {peak }}(W) ;(B): P_{\text {peak }}\left(W \cdot \mathrm{kg}^{-0.67)}\right)$; (C): $P_{\text {mean }}(W) ;(D): P_{\text {mean }}\left(W \cdot \mathrm{kg}^{-0.67}\right),(E): F I\left(W \cdot \mathrm{s}^{-1}\right)$.

The TAIKT was considered to have very good discriminant validity. The area under the ROC curve (AUC) was 0.93 (95\%CI: 0.72-0.99, $p<$ 0.0001) for P PeakTAIKT (W) (Figure 2A), 0.94 (95\%CI: $0.74-0.99, \quad p<0.0001)$ for P PeakTAIKT $\left(\mathrm{W} \cdot \mathrm{kg}^{-0.67}\right)$ (Figure 2B), 0.96 (95\%CI: 0.75-0.99, $p<0.0001$ ) for PmeantaIKT (W) (Figure 2C) and 0.93 (95\%CI: 0.72$0.99, p<0.0001)$ for PmeantaIкт $\left(\mathrm{W} \cdot \mathrm{kg}^{-0.67}\right)$ (Figure 2D). The AUC of FItaikt was 0.79 (95\%CI: 0.540.94, $p=0.0093$ ) (Figure 2E).

\section{Discussion}

The main purpose of the TAIKT is to assess the specific anaerobic power of taekwondo athletes while reproducing the intermittent effort exerted during taekwondo combat. It has previously been established that the TAIKT is a valid and reliable sport-specific test (Tayech et al., 2018). The aims of the current research were (i) to examine absolute and relative reliability of the WAnT, SJ, and CMJ, validity of the TAIKT 
compared with the WAnT, SJ and CMJ tests, and (ii) to verify its ability to effectively distinguish (i.e., construct validity) between taekwondo athletes of different competitive levels (i.e., elite and sub-elite). The main findings showed that the WAnT, SJ, and CMJ presented good relative and absolute reliability among taekwondo athletes. Moreover, significant large correlations between the TAIKT and the WAnT, SJ, and CMJ were found. Thus, the TAIKT may be considered a sport-specific field test able to distinguish between elite and sub-elite Taekwondo athletes.

According to Impellizzeri and Marcora (2009), reliability results are most often used to assess whether a physical fitness test protocol should be widely used. Against this background, reliability results from the current study showed that all WAnT, SJ, and CMJ performances, as well as physiological and perceptual variables, displayed almost excellent relative and absolute reliability. An excellent ICC was found $(>0.90)$ (Driss and Vandewalle, 2013; Hopkins, 2009) in conjunction with a small SEM, in the variables determined from the WAnT, SJ and CMJ (i.e., absolute and relative $\mathrm{P}_{\text {peakWAnT }}$ and $\mathrm{P}_{\text {meanWAnT, }} \mathrm{P}_{\text {peakS }}$ and $\mathrm{P}_{\text {peakCMI}}$ ), and good relative reliability for FIWAnT $(I C C=0.82)$. In accordance with this last result, Driss and Vandewalle (2013) reported that the FI was the least reliable of the three WAnT indices. Consequently, the authors claimed that $P_{\text {peak }}$ and $P_{\text {mean }}$ output of the WAnT were the main topics of most studies examining the reliability and validity of new field anaerobic tests. The same results were established at good absolute and relative levels for HReakWAnT (ICC $=0.71$ ) and RPEWAnT (ICC $=0.82$ ). The ability to detect small performance changes supported by values of SWC was good, which presents an SWC value slightly above the SEM, with SEM far below the $5 \%$ limit, except for FIwAnT, HRpeakWAnT, and RPEWAnT, which presented a SWC lower than SEM, and was rated as marginal (Liow and Hopkins, 2003). The minimal detectable change (MDC95\%) of tests which represented the 95\% CI of the difference in scores between paired observations was acceptable for most of the variables. One of the most important factors that supported the stability of the reliability of tests (i.e., WAnT, SJ, and CMJ) was the method of Bland and Altman plots, which presented excellent results of concordance in all variables.
Indeed, Hopkins (2000) reported that this measure of absolute reliability was too stringent to determine whether a change in an individual's score was real or was an artefact of measurement error.

The WAnT is widely accepted as a valid "Gold standard" anaerobic test (Bar-Or, 1987; Driss and Vandewalle, 2013). It has been much more extensively used than any other anaerobic test (Driss and Vandewalle, 2013). Findings of this study showed moderate-to-large correlation coefficients between the TAIKT and the WAnT in all performance (i.e., $P_{\text {peak, }} P_{\text {mean, }}$ and FI), physiological (i.e., HReak and [La-]) and perceptual (i.e., RPE) variables. This correlation between the $P_{\text {peak }}$ and $P_{\text {mean }}$ values of both tests (i.e., TAIKT and WAnT) may provide useful estimates of anaerobic power relative to the TAIKT (Sands et al., 2004). In terms of physiological variables, evidence of the anaerobic nature of the TAIKT has been supported by the magnitude of the correlation coefficient $(r=0.53 ; p$ $<0.05$ ) between the TAIKT and the WAnT in [ $\left.\mathrm{La}^{-}\right]$

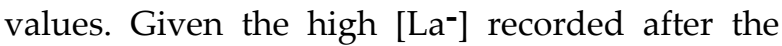
end of the TAIKT, it seems plausible to argue that anaerobic glycolysis dominated in provision of

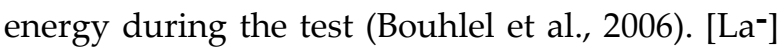
concentrations after the WAnT performance have not previously been reported in taekwondo athletes (Bridge et al., 2014). Accordingly, [La-] values observed following the WAnT $(11.05 \pm 2.17$ mmol. $\mathrm{l}^{-1}$ ) are comparable to those recorded following the TAIKT $\left(10.8 \pm 1.6 \mathrm{mmol} \cdot \mathrm{l}^{-1}\right)$. Additionally, [ $\left.\mathrm{La}^{-}\right]$values observed following the TAIKT are similar to the values recorded at the end of an official taekwondo combat (Bridge et al., 2009; Markovic et al., 2008). Bouhlel et al. (2006) confirmed that the significant rise of [ $\left.\mathrm{La}^{-}\right]$ concentrations during competition indicated that the glycolytic pathway (Facey et al., 2013) was also important in Taekwondo bouts, especially during repetitive high-intensity actions (e.g., attack and counter-attack sequences). Furthermore, in the current study, a large significant correlation was observed in $\mathrm{HR}_{\text {peak }}$ between the two anaerobic tests (i.e., TAIKT and WAnT). HR $_{\text {peakTAIKT }}(\approx 188 \mathrm{bpm})$ and HRpeakWAnT $(\approx$ $187 \mathrm{bpm})$ were close the age-predicted maximal HR $(\approx 90 \%)$. This result is in agreement with the study of Bouhlel et al. (2006), who claimed that HRs observed during 10-s and 1-min specific 
taekwondo exercises were about $91 \%$ and $92 \%$, respectively, of $\mathrm{HR}_{\text {peak }}$ measured during simulated competition. Hence, the high HRpeakWAnT and [ $\mathrm{La}^{-}$ тАाкт] values clearly pointed to the maximal character of the TAIKT with similar values as those seen during taekwondo competition (Bouhlel et al., 2006). In terms of internal load assessment, the subjective perception of effort (i.e., RPE) has been shown to be a valid approach to quantify effort's intensity during taekwondo training (Haddad et al., 2011, 2014; Perandini et al., 2012) and competition (Bridge et al., 2009; Haddad et al., 2011; Markovic et al., 2008). Findings of this study showed a large correlation $(r=0.55, p<0.05)$ between RPE values derived from the TAIKT and the WAnT.

As power and accuracy of each blow are crucial to effectively score (i.e., Protector Scoring System (PSS)) (Del Vecchio et al., 2011), the lower limb muscle power is a variable of interest to evaluate the muscular mechanical characteristics of taekwondo athletes (Rocha et al., 2016a). Once expressing TAIKT performance (i.e., $\left.P_{\text {peak}}\right)$ allometrically (i.e., $\mathrm{W} \cdot \mathrm{kg}^{-0.67}$ ) (Chamari et al., 2008), large correlation coefficients were observed with SJ $(r=0.60, p<0.01)$ and CMJ $(r=0.63, p<0.01)$ tests. Therefore, taekwondo athletes may benefit from the TAIKT as a valid field sport-specific test assessing anaerobic power, heavily reliant on explosive actions of the lower limbs as in taekwondo competition.

One of the most important objectives of this study was to evaluate the construct validity (sensitivity) of the TAIKT that is its ability to discriminate between taekwondo athletes of different competitive levels. Against this background, Impellizzeri and Marcora (2009) suggested using the ROC approach to examine the discriminative power and the responsiveness of performance tests. Similarly to the recent study of Chaabene et al. (2018) conducted with Tunisian elite taekwondo athletes, the ROC analysis was used to determine the ability of the TAIKT to discriminate between elite and sub-elite athletes. In this regard, significant differences between TAIKT's performances of elite and sub-elite athletes in favour of the elite were observed. The area under the ROC curve (AUC), which represents the probability of correctly discriminating between elite and sub-elite taekwondo athletes using the TAIKT (Deyo and
Centor, 1986; Mannion et al., 2005) was $>0.90$ for absolute and relative P PeakTAIKт and PmeanTAIKT, respectively. These scores are considered very close to complete accuracy (De Vet et al., 2001). The AUC of FI was 0.79, which is commonly considered to indicate good discriminative ability (Deyo and Centor, 1986; Mannion et al., 2005). The test's scores able to differentiate between elite and sub-elite taekwondo athletes are $>15.18 \mathrm{~W}$ and $>0.86 \mathrm{~W} \cdot \mathrm{kg}^{-67}$ for P PeakTAIKT, $>9.59 \mathrm{~W}$ and $>0.59$ $\mathrm{W} \cdot \mathrm{kg}^{-67}$ for PmeantaikT, and $>0.32 \mathrm{~W} \cdot \mathrm{s}^{-1}$ for FІталкт. These cut-off values provide a true positive rate (sensitivity) range between $70 \%$ and $90 \%$ and a false positive rate (1-specificity) range between $89 \%$ and $100 \%$. In addition, with the results demonstrated in this study, coaches could use the TAIKT as an appropriate tool to distinguish between taekwondo athletes of various competitive levels. Nevertheless, no significant differences were found between elite and sub-elite taekwondo athletes in physiological (i.e.,

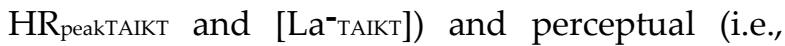
RPEтAIкT) variables. This is in line with findings of Jones and Unnithan (1998) in Kung-fu practitioners and Chaabène et al. (2012) in karate athletes. A limitation of the present study is that in the experiment the sample included two levels of elite athletes only (i.e., elite and sub-elite) and a limited number of female athletes (i.e., 5 females). Therefore, further studies are required to verify the validity and reliability of the TAIKT among taekwondo athletes of different age, gender and athletic level. Despite this limit, the present findings showed that the TAIKT is a sport-specific kicking test that may be a more specific and easy measure of anaerobic power among taekwondo athletes than the other previously proposed taekwondo tests. Furthermore, given that the TAIKT is a non-invasive, easy to perform and accessible test, it can be regularly used by coaches throughout the season to evaluate taekwondo athletes' sport-specific anaerobic performance (i.e., intermittent high-intensity power). In conclusion, the main outcomes of this study indicate that the TAIKT is a valid test characterized by a good ability to discriminate between elite and sub-elite taekwondo athletes. Most importantly, the internal training load outcomes indicate that TAIKT's activity could mirror Taekwondo combat effort. 


\section{Acknowledgements}

The authors are grateful to all the participants of the Tunisian National Taekwondo Team for their valuable time and contribution. We thank Mr. Mohamed Ali Rouahi, Mr. Seifeddine Trabelsi and Mr. Anouar Jlassi for their help in the experimentation. This study was supported by The Research Laboratory "Sports Performance Optimization" National Center of Medicine and Science in Sports (CNMSS).

\section{References}

Araujo MP, Nóbrega AC, Espinosa G, Hausen MR, Castro RR, Soares PP, Gurgel JL. Proposal of a new specific cardiopulmonary exercise test for Taekwondo athletes. J Strength Cond Res, 2017; 31: 15251535

Attia A, Hachana Y, Chaabène H, Gaddour A, Neji Z, Shephard RJ, Chelly, MS. Reliability and validity of a 20-s alternative to the wingate anaerobic test in team sport male athletes. PloS one, 2014; 9: e114444

Bar-Or O. The Wingate anaerobic test: An update on methodology, reliability, and validity. Sports Med, 1987; 4: 381-394

Bland JM, Altman DG. Statistical methods for assessing agreement between two methods of clinical measurement. Lancet, 1986; 1: 307-310

Bouhlel E, Jouini A, Gmada N, Nefzi A, Abdallah KB, Tabka Z. Heart rate and blood lactate responses during Taekwondo training and competition. Sci Sport, 2006; 2: 285-290

Bridge CA, Ferreira da Silva Santos J, Chaabène H, Pieter W, Franchini E. Physical and physiological profiles of taekwondo athletes. Sports Med, 2014; 44: 713-733

Bridge CA, Jones MA, Drust B. Physiological responses and perceived exertion during international taekwondo competition. Int J Sports Physiol Perform, 2009; 4: 485-493

Chaabène H, Hachana Y, Franchini E, Mkaouer B, Montassar M, Chamari K. Reliability and construct validity of the karate-specific aerobic test. J Strength Cond Res, 2012; 26: 3454-3460

Chaabene H, Negra Y, Capranica L, Bouguezzi R, Hachana Y, Rouahi MA, Mkaouer B. Validity and reliability of a new test of planned agility in elite taekwondo athletes. J Strength Cond Res, 2018; Epub ahead of print

Chamari K, Chaouachi A, Hambli M, Kaouech F, Wisløff U, Castagna C. The five-jump test for distance as a field test to assess lower limb explosive power in soccer players. J Strength Cond Res, 2008; 22: 944-950

Chaouachi A, Castagna C, Chtara M, Brughelli M, Turki O, Galy O, Chamari K, Behm DG. Effect of warmups involving static or dynamic stretching on agility, sprinting, and jumping performance in trained individuals. J Strength Cond Res, 2010; 24: 2001-2011

Chaouachi A, Padulo J, Kasmi S, Othmen AB, Chatra M, Behm, DG. Unilateral static and dynamic hamstrings stretching increases contralateral hip flexion range of motion. Clin Physiol Funct Imaging, 2017; 37: 23-29

De Vet HC, Bouter LM, Bezemer PD, Beurskens AJ. Reproducibility and responsiveness of evaluative outcome measures. Theoretical considerations illustrated by an empirical example. Int J Technol Assess Health Care, 2001; 17: 479-487

Del Vecchio FB, Franchini E, Del Vecchio AHM, Pieter W. Energy absorbed by electronic body protectors from kicks in a taekwondo competition. Biol Sport, 2011; 28: 75-78

Deyo RA, Centor, RM. Assessing the responsiveness of functional scales to clinical change: An analogy to diagnostic test performance. J Chronic Dis, 1986; 39: 897-906

Driss T, Vandewalle H. The measurement of maximal (anaerobic) power output on a cycle ergometer: a critical review. Biomed Res Int, 2013

Facey A, Irving R, Dilworth L. Overview of lactate metabolism and the implications for athletes. Am J Sport Sci Med, 2013; 1: 42-46

Faul F, Erdfelder E. GPOWER: A Priori, Post-Hoc, and Compromise Power Analyses for MS-DOS (Computer Program). Bonn, FRG. Bonn University: Department of Psychology; 2004 
Hachana Y, Attia A, Nassib S, Shephard RJ, Chelly MS. Test-retest reliability, criterion-related validity, and minimal detectable change of score on an abbreviated Wingate test for field sport participants. $J$ Strength Cond Res, 2012; 26: 1324-1330

Haddad M, Chaouachi A, Castagna C, Wong DP, Behm DG, Chamari K. The construct validity of session RPE during an intensive camp in young male Taekwondo athletes. Int J Sports Physiol Perform, 2011; 6: 252-263

Haddad M, Chaouachi A, Wong DP, Castagna C, Hue O, Impellizzeri FM, Chamari K. Influence of exercise intensity and duration on perceived exertion in adolescent Taekwondo athletes. Eur J Sport Sci, 2014; 14: S275-S281

Hopkins WG. A scale of magnitudes for effect statistics. SportScience, 2009. Available at: http://www.sportsci.org/resource/stats/index.html

Hopkins WG. How to interpret changes in an athletic performance test. Sportscience, 2004; 8: 1-7.

Hopkins WG. Measures of reliability in sports medicine and science. Sports Med, 2000; 30: 1-15.

Impellizzeri FM, Marcora SM. Test validation in sport physiology: Lessons learned from clinimetrics. Int J Sports Physiol Perform, 2009; 4: 269-277

Jones MA, Unnithan VB. The cardiovascular responses of male subjects to kung fu techniques. J Sports Med Phys Fitness, 1998; 38: 323-329

Liow DK, Hopkins WG. Velocity specificity of weight training for kayak sprint performance. Med Sci Sports Exerc, 2003; 35: 1232-1237

Mannion AF, Elfering A, Staerkle R, Junge A, Grob D, Semmer NK, Jacobshagen N, Dvorak J, Boos N. Outcome assessment in low back pain: how low can you go? Eur Spine, 2005; J14: 1014-1026

Markovic G, Vucetic V, Cardinale M. Heart rate and lactate responses to taekwondo fight in elite women performers. Biol Sport, 2008; 25: 135-146

Perandini LA, Siqueira-Pereira TA, Okuno NM, Soares-Caldeira LF, Nakamura FY. Use of session RPE to training load quantification and training intensity distribution in taekwondo athletes. Sci Sports, 2012; 27: e25-e30

Rocha F, Louro H, Matias R, Costa A. Anaerobic fitness assessment in taekwondo athletes. A new perspective. Motricidade, 2016a; 12: 127-139

Rocha FP, Louro H, Matias R, Brito J, Costa AM. Determination of Aerobic Power Through a Specific Test for Taekwondo-A Predictive Equation Model. J Hum Kinet, 2016b; 53: 117-126

Sands WA, McNeal JR, Ochi MT, Urbanek TL, Jemni M, Stone MH. Comparison of the Wingate and Bosco anaerobic tests. J Strength Cond Res, 2004; 18: 810-815

Sant'Ana J, Diefenthaeler F, Dal Pupo J, Detanico D, Guglielmo LGA, Santos, SG. Anaerobic evaluation of Taekwondo athletes: original research. IntSportmed J, 2014; 15: 492-499

Stratford PW. Getting more from the literature: estimating the standard error of measurement from reliability studies. Physiother Can, 2004; 56: 27-30

Tayech A, Mejri MA, Chaabene H, Chaouachi M, Behm DG, Chaouachi A. Test-retest reliability and criterion validity of a new Taekwondo anaerobic intermittent kick test. J Sports Med Phys Fitness, 2018; Epub ahead of print

World Taekwondo. http://www.worldtaekwondo.org/?s=scientific. 2018

\section{Corresponding author:}

Dr. Mohamed Arbi Mejri,

Tunisian Research Laboratory "Sports Performance Optimization", National Center of Medicine and Science in Sports (CNMSS), Tunis, Tunisia. Ave Med Ali Akid, 1004 El Menzah, Tunis, BP263, Tunisia. Phone: + 216 55731648; Fax: + 216 70709789;

E-mail: mejriarbi@gmail.com 